Wouter I. Schievink

\author{
M. M. Maya \\ Franklin Moser \\ James Tourje \\ Sam Torbati
}

\section{Frequency of spontaneous intracranial hypotension in the emergency department}

Received: 6 September 2007

Accepted in revised form: 1 October 2007

Published online: 17 December 2007

W.I. Schievink ( $\square)$

Department of Neurosurgery,

The Maxine Dunitz Neurosurgical Institute, Cedars-Sinai Medical Center, 8631 West Third Street, Suite 800E, Los Angeles, CA 90048, USA

e-mail: schievinkw@cshs.org

Tel.: +1-310-423-7900

Fax: +1-310-423-0810

M.M. Maya • F. Moser • J. Tourje

Imaging Medical Group,

Cedars-Sinai Medical Center,

Los Angeles, CA, USA

S. Torbati

Department of Emergency Medicine,

Cedars-Sinai Medical Center,

Los Angeles, CA, USA

\begin{abstract}
Spontaneous intracranial hypotension is considered a rare disorder. We conducted a study on the frequency of spontaneous intracranial hypotension in the emergency department (ED). We identified patients with spontaneous intracranial hypotension evaluated in the ED of a large urban hospital between 1 January 2003 and 31 December 2006. For comparison, we also identified all patients with spontaneous subarachnoid haemorrhage (SAH). Eleven patients with previously undiagnosed spontaneous intracranial hypotension were evaluated in the ED during the four-year time period. All patients presented with positional headaches and the duration of symptoms varied from one day to three months. None of the patients were correctly diagnosed with spontaneous intracranial
\end{abstract}

\begin{abstract}
hypotension in the ED. During the same time period, 23 patients with aneurysmal SAH were evaluated. Spontaneous intracranial hypotension is more common than previously appreciated and the diagnosis in the ED remains problematic.
\end{abstract}

Keywords Emergency medicine • Epidemiology $\cdot$ Headache • Intracranial hypotension

\section{Introduction}

Spontaneous intracranial hypotension is an important cause of new daily persistent headaches, particularly among young and middle-aged adults [1]. Spontaneous intracranial hypotension is caused by a spontaneous spinal cerebrospinal fluid (CSF) leak, often in the setting of an underlying generalised connective tissue disorder [1]. The clinical hallmark of spontaneous intracranial hypotension is the presence of a headache that is exacerbated by the upright position and relieved by ***recumbancy. Such a characteristic positional component is present, at least initially, in the great majority of patients but misdiagnosis remains common [2]. The headache of spontaneous 
intracranial hypotension is not always orthostatic and various other headache patterns have been described. The onset of headache may be acute, mimicking a subarachnoid haemorrhage (SAH) [1]. In addition to headache, a wide variety of associated symptoms has been reported, such as neck pain, photo- or phonophobia, diplopia, parkinsonism and even coma. This varied symptomatology contributes to the diagnostic difficulties. Typical imaging features on computed tomography (CT) or magnetic resonance imaging (MRI) also may contribute to a diagnostic challenge with sagging of the brain mimicking a Chiari malformation, enhancement of the pachymeninges mimicking infectious or neoplastic disease, subdural fluid collections mimicking a primary subdural haematoma, and pituitary hyperaemia mimicking a pituitary tumour [1]. Because opening pressure generally is low in these patients, a traumatic tap is common, further causing confusion with SAH [1]. Treatment for the underlying spontaneous spinal CSF leak includes bed rest and hydration, epidural blood patching, percutaneous injection of fibrin glue and surgical repair [1].

Spontaneous intracranial hypotension generally is considered to be a rare, albeit underdiagnosed, disorder and no epidemiologic studies have been reported. We now report a study on the frequency of spontaneous intracranial hypotension in the emergency department (ED), demonstrating that spontaneous intracranial hypotension is much more common than previously appreciated.

\section{Materials and methods}

In January 2002, we began a four-year observational study of spontaneous intracranial hypotension in the ED of a large urban hospital (Cedars-Sinai Medical Center) in Los Angeles, California, with the ED seeing approximately 60000 patient visits per year. Cases were ascertained through a review of all neuroradiological studies compatible with spontaneous intracranial hypotension among patients evaluated in the ED. The ED notes were reviewed for the purpose of this study. The diagnosis of spontaneous intracranial hypotension was confirmed by CTmyelography showing a CSF leak in all patients. Only patients with previously undiagnosed spontaneous intracranial hypotension were included in the study. All patients were seen by one of us in consultation after the diagnosis of spontaneous intracranial hypotension had been established.

In order to compare the frequency of spontaneous intracranial hypotension with a well recognised neurologic disorder that has a well established incidence rate, we also identified all patients seen in our ED with SAH during the same time period. Patients who were transferred from another facility were excluded from the analysis.

\section{Results}

Between 1 January 2002 and 31 December 2006, 11 patients with previously undiagnosed spontaneous intracranial hypotension were evaluated in our ED (Table 1 ). The mean age of the seven women and four men was 38.3 years (range, 22-54 years). All patients resided in Los Angeles County within 20 miles of our medical centre. All patients presented with positional headaches and the duration of symptoms varied from one day to three months. In two patients, the positional nature of the headache was reflected in the ED note. None of the patients were diagnosed with spontaneous intracranial hypotension in the ED.

Six patients $(55 \%)$ had abnormal CT findings consisting of subdural fluid collections $(n=4)$, cerebellar tonsillar herniation $(n=1)$ or obliteration of the basilar cisterns $(n=1)$. These were considered to be the primary abnormalities in the ED and the patients were admitted to hospital. Upon further review of the CT by the attending neuroradiologists or neurosurgeon, a diagnosis of probable spontaneous intracranial hypotension was made and further imaging confirmed the diagnosis. Because of the severity of the headache, all five patients with normal CT results underwent a lumbar puncture in the ED. A formal opening pressure was obtained in one patient who had a borderline low pressure $(7 \mathrm{~cm} \mathrm{H} 2 \mathrm{O})$. In the remaining four patients, the lumbar punctures were described as "very traumatic and unable to obtain more than $2 \mathrm{cc}$ of fluid" in one patient, as "CSF draining so slowly that the opening pressure could not be measured" in one patient, as a "dry tap on multiple attempts" in one patient, and as "uneventful" in one patient. These findings were not considered significant in any of these five patients. Eventually, spontaneous intracranial hypotension was diagnosed on the basis of abnormal MRI findings (i.e., pachymeningeal enhancement and brain sagging).

A variety of treatments were necessary to achieve satisfactory relief of symptoms, including (in order of invasiveness) bed rest and hydration in one patient, epidural blood patching in eight patients, percutaneous injection of a fibrin sealant in one patient and surgical CSF leak repair in one patient. One patient also underwent burr-hole drainage of an associated subdural haematoma.

During the four-year study period, 23 patients with aneurysmal SAH were evaluated in our ED.

\section{Discussion}

In this study of patients evaluated at a large urban ED, spontaneous intracranial hypotension was not rare and it 
was diagnosed about half as frequently as aneurysmal SAH. The incidence of aneurysmal SAH in the USA is approximately 10 per 100,000 per year [3], suggesting an annual incidence of 5 per 100,000 for spontaneous intracranial hypotension. However, this was not a communitybased study and the incidence figure is only a gross approximation. Prior studies on the epidemiology of spontaneous intracranial hypotension are not available for comparison, with the exception of one unpublished study identifying two patients with spontaneous intracranial hypotension in a community of approximately 100,000 persons in southeastern Minnesota for a prevalence of approximately 1 in 50,000 [4]. The frequency of spontaneous intracranial hypotension in our ED was likely an underestimation because we primarily used abnormal CT scanning to identify patients with spontaneous intracranial hypotension and no special effort was made to identify patients with spontaneous intracranial hypotension based on clinical characteristics. Thus, patients with spontaneous intracranial hypotension and normal CT results were not included in our study, unless subsequent MRI or CT-myelography showing spontaneous intracranial hypotension was performed in our institution or was otherwise brought to our attention. Headache is a fairly common presenting complaint in the ED, accounting for approximately $0.5 \%-2 \%$ of all ED visits [5-9]. Among the approximately 240000 visits to our ED during the four-year period of our study, it can be estimated that headache was the primary complaint in
1200-4800 patients and that spontaneous intracranial hypotension accounted for approximately $0.25 \%-1 \%$ of headaches. Thus, among all patients with headache seen in the ED, spontaneous intracranial hypotension is an infrequently diagnosed disorder.

The symptomatology among the patients in this study was typical of spontaneous intracranial hypotension with a positional headache present in all patients. Nevertheless, the positional nature of the headache was noted in only two of the ED reports and, similar to previous studies, the diagnosis of spontaneous intracranial hypotension was not considered in the ED in any of the patients. Head CT was abnormal in more than half of the patients and the imaging abnormalities (subdural haematoma, Chiari malformation and "cerebral oedema") were considered to be the primary diagnoses. In those patients with normal CT results, the low opening pressure on lumbar puncture was not recognised as abnormal and no specific headache diagnosis was made.

The main limitation of this study was its retrospective nature and it is likely that patients with spontaneous intracranial hypotension went unrecognised. However, the frequency of spontaneous intracranial hypotension was much higher than expected based on previous reports of spontaneous intracranial hypotension. Most of the limitations associated with retrospective studies would likely result in an underestimation, rather than an overestimation, of the frequency of spontaneous intracranial hypotension. Our study is also limited by the nature of its use of an ED

Table 1 Clinical and radiologic characteristics of 11 patients with spontaneous intracranial hypotension presenting to the ED in $2003-2006$

\begin{tabular}{|c|c|c|c|c|c|c|}
\hline No. & Date & Age, sex & $\begin{array}{l}\text { Duration } \\
\text { of symptoms }\end{array}$ & $\begin{array}{l}\text { Initial CT } \\
\text { diagnosis }\end{array}$ & $\begin{array}{l}\text { Site of } \\
\text { CSF leak }\end{array}$ & Treatment \\
\hline 1 & $5 / 2003$ & $39, \mathrm{~F}$ & $\sim 3$ weeks & Subdural haematoma & Cervical & $\begin{array}{l}\text { Epidural blood patch, } \\
\text { percutaneous fibrin } \\
\text { sealant placement, } \\
\text { surgical CSF leak repair }\end{array}$ \\
\hline 2 & $6 / 2003$ & $28, \mathrm{M}$ & 25 days & Subdural haematoma & Cervical & $\begin{array}{l}\text { Epidural blood patch, } \\
\text { percutaneous fibrin } \\
\text { sealant placement }\end{array}$ \\
\hline 3 & $1 / 2004$ & $50, \mathrm{~F}$ & 1 day & Normal & Thoracic & Bed rest \\
\hline 4 & $1 / 2004$ & $54, \mathrm{~F}$ & 1 day & Cerebral oedema & Thoracic & Epidural blood patch \\
\hline 5 & $5 / 2004$ & $41, \mathrm{~F}$ & 3 days & Normal & Thoracic & Epidural blood patch \\
\hline 6 & $11 / 2004$ & $22, \mathrm{~F}$ & $\sim 2$ weeks & Chiari malformation & Thoracic & Epidural blood patch \\
\hline 7 & $2 / 2005$ & $50, \mathrm{M}$ & 7 days & Subdural haematoma & Thoracic & $\begin{array}{l}\text { Burr-hole drainage and } \\
\text { epidural blood patch }\end{array}$ \\
\hline 8 & $5 / 2005$ & $26, \mathrm{M}$ & 3 months & Subdural haematoma & Thoracic & Epidural blood patch \\
\hline 9 & $2 / 2006$ & $51, \mathrm{~F}$ & 3 days & Normal & Cervico-thoracic & Epidural blood patch \\
\hline 10 & $6 / 2006$ & $29, \mathrm{~F}$ & 4 days & Normal & Thoraco-lumbar & Epidural blood patch \\
\hline 11 & $7 / 2006$ & $31, \mathrm{M}$ & 1 day & Normal & Thoracic & Epidural blood patch \\
\hline
\end{tabular}


setting. Although most patients with spontaneous intracranial hypotension visit an ED during the course of their illness [2], this is not a universal occurrence, affecting its comparison to the incidence of SAH. An additional limitation is that not all patients with a main complaint of headache were identified, affecting the estimate of spontaneous intracranial hypotension among all patients with headache in the ED. Finally, our analysis is restricted to one large urban hospital in Southern California, USA, and generalisability of our findings is uncertain. In retrospect, inclusion of other medical facilities would have been warranted based on the rela- tively frequent occurrence of spontaneous intracranial hypotension in the ED. However, this information was not available at the time the study was initiated.

\section{Conclusion}

Spontaneous intracranial hypotension is much more common than previously appreciated and its diagnosis in the ED remains problematic.

\section{References}

1. Schievink WI (2006) Spontaneous spinal cerebrospinal fluid leaks and intracranial hypotension. JAMA 295: 2286-2296

2. Schievink WI (2003) Misdiagnosis of spontaneous intracranial hypotension. Arch Neurology 60:1713-1738

3. Brown RD, Whisnant JP, Sicks JD, et al (1996) Stroke incidence, prevalence, and survival: secular trends in Rochester, Minnesota, through 1989. Stroke 27:370-372

4. Schievink WI, Morreale VM, Atkinson JL et al (1998) Surgical treatment of spontaneous spinal cerebrospinal fluid leaks. J Neurosurg 88:243-246
5. Leicht M (1980) Non-traumatic headache in the emergency department. Ann Emerg Med 9:404-409

6. Barton CW (1994) Evaluation and treatment of headache patients in the emergency department: A survey. Headache 34:91-94

7. Morgenstern LB, Huber JC, LunaGonzales H et al (2001) Headache in the Emergency Department. Headache 41:537-541
8. Locker T, Mason S, Rigby A (2004) Headache management-Are we doing enough? An observational study of patients presenting with headache to the emergency department. Emerg Med J 21:327-332

9. Goldstein JN, Camargo CA Jr, Pelletier AJ, Edlow JA (2006) Headache in United States emergency departments: demographics, work-up and frequency of pathological diagnoses. Cephalalgia 26:684-690 